\title{
Classical bremsstrahlung radiation from electron-ion encounters in a nonideal plasma
}

\author{
Y O U N G-D A E J U N G G,2 and C H A N G-G E U N K I M ${ }^{2}$ \\ ${ }^{1}$ Department of Physics, 0319, University of California, San Diego, 9500 Gilman Drive, \\ La Jolla, CA 92093-0319, USA \\ ${ }^{2}$ Department of Physies, Hanyang University, Ansan, Kyunggi-Do 425-791, South Korea \\ (ydjung@physics.ucsd.edu; yjung@bohr.hanyang.ac.kr)
}

(Received 18 October 2001)

\begin{abstract}
The classical electron-ion Coulomb bremsstrahlung process is investigated in a nonideal plasma. An effective pseudopotential model taking into account plasma-screening and collective effects is applied to describe the electron-ion interaction potential in a nonideal plasma. The screened hyperbolic-orbit trajectory method is applied to the motion of the projectile electron in order to investigate the bremsstrahlung radiation cross-section as a function of the scaled impact parameter, eccentricity, nonideal-plasma parameter, Debye length, projectile energy, and photon energy. It is found that the collective effect reduces the bremsstrahlung radiation cross-section on both the soft- and hard-photon cases. For small impact parameters, the nonideal-plasma effect on the bremsstrahlung radiation cross-section is found to be quite small. It is also found that the maximum position of the bremsstrahlung radiation cross-section gets closer to the target ion with increasing nonideal-plasma effect.
\end{abstract}

\section{Introduction}

Bremsstrahlung processes (Bethe and Salpeter 1957; Bekefi 1966; Gould 1970, 1980, 1990; Hutchinson 1987; Jung 1994, 1997; Tsytovich 1995) in a plasma have received much attention, since these processes are widely used in plasma diagnostics in laboratory and astrophysical plasmas. Recently, bremsstrahlung processes in a weakly coupled plasma (Gould 1990; Jung 1997) have been investigated using the Debye-Hückel model (Jung and Yang 1997; Padmanabhan 2001) potential with the classical-trajectory method. The Debye-Hückel effective potential describes the properties of a low-density plasma, and corresponds to a pair-correlation approximation. A plasma described by the Debye-Hückel model is known as an ideal plasma, since the average energy of interaction between particles is small compared with the average kinetic energy of a particle. However, multiparticle correlation effects caused by simultaneous interaction of many particles should be taken into account with increasing plasma density. It is necessary to take into account not only short-range collective effects but also long-range effects in the case of a plasma with a moderate density and temperature. In this case, the interaction potential is different from the Debye-Hückel type because of the strong collective effects of nonideal particle interaction. Then, the bremsstrahlung spectrum from 
the electron-ion Coulomb scatterings in a nonideal plasma must be quite different from that in an ideal plasma because of the collective effect. Therefore, in this paper, we investigate the classical bremsstrahlung process due to electron-ion collisions in a nonideal plasma. A pseudopotential model including plasma-screening and collective effects is applied to describe the interaction potential between the projectile electron and target ion in a nonideal plasma. The hyperbolic-orbit trajectory method (Landau and Lifshitz 1975; Jung and Yang 1997) is applied to represent the motion of the projectile electron and to visualize the bremsstrahlung radiation cross-section as a function of the scaled impact parameter, eccentricity, nonideal-plasma parameter, Debye length, projectile energy, and radiation photon energy.

In Sec. 2, we discuss the classical expression for the bremsstrahlung cross-section in Coulomb scattering of the projectile electron with the target ion in a nonideal plasma using the pseudopotential model. In Sec. 3, we obtain an analytical form of the bremsstrahlung radiation cross-section using the screened hyperbolic-orbit trajectory method for the motion of the projectile electron. We also investigate the variation of the bremsstrahlung radiation cross-sections for both the soft- and hard-photon radiation cases with changing impact parameter and nonideal-plasma parameter. Finally, conclusions are given in Sec. 4.

\section{Classical bremsstrahlung cross-section}

The cross-section for the classical bremsstrahlung process (Gould 1970) is given by

$$
d \sigma_{b}=2 \pi \int d b b d w_{\omega}(b)
$$

where $b$ is the impact parameter and $d w_{\omega}$ is the differential probability of emitting a photon of frequency $\omega$ within $d \omega$ when the projectile electron changes its velocity in collisions with a static target system. For all impact parameters, the radiation probability $d w_{\omega}$, can be obtained by the Larmor formula (Jackson 1999) for the emission spectrum of a nonrelativistic accelerated electron:

$$
d w_{\omega}=\frac{8 \pi e^{2}}{3 \hbar c^{3}}\left|\mathbf{a}_{\omega}\right|^{2} \frac{d \omega}{\omega},
$$

where $\mathbf{a}_{\omega}$ is the Fourier coefficient of the acceleration $\mathbf{a}(t)$ of the projectile electron:

$$
\mathbf{a}_{\omega}=\frac{1}{2 \pi} \int_{-\infty}^{\infty} d t e^{i \omega t} \mathbf{a}(t) .
$$

Here, we set up coordinate axes to compute $\mathbf{a}_{\omega}$ so that the electron trajectory is in the $(x, y)$ plane; then

$$
\left|\mathbf{a}_{\omega}\right|^{2}=\frac{1}{m^{2}}\left(\left|F_{x \omega}\right|^{2}+\left|F_{y \omega}\right|^{2}\right),
$$

where $F_{x \omega}$, and $F_{y \omega}$ are, respectively, the $x$ and $y$ components of the Fourier coefficients of the force $\mathbf{F}(t)$ between the projectile electron and the target ion:

$$
F_{\mu \omega}=\frac{1}{2 \pi} \int_{-\infty}^{\infty} d t e^{i \omega t} F_{\mu}(t) \quad(\mu=x, y) .
$$

An integro-differential equation for the effective potential of the particle interaction, taking into account the simultaneous correlations of $N$ particles, has been 
obtained on the basis of a sequential solution of the chain of Bogolyubov equations for the equilibrium distribution function of particles of a classical nonideal plasma, and an analytical expression for the pseudopotential (Baimbetov et al. 1995) of the particle interaction in a nonideal plasma has been obtained by application of the spline approximation. Using the pseudopotential model, taking into account plasma-screening and collective effects (Arkhipov et al. 2000a,b, 2001), the interaction potential $V_{N I}(\mathbf{r})$ between the projectile electron and the target ion with charge $Z$ in a classical nonideal plasma can be written as

$$
V_{N I}(\mathbf{r})=-\frac{Z e^{2}}{r} e^{-r / \Lambda} \frac{1+\frac{1}{2} \gamma f(\gamma, r, \Lambda)}{1+c(\gamma)},
$$

where $\mathbf{r}$ is the position vector of the projectile electron with respect to the target ion, $\Lambda$ is the Debye length, $f(\gamma, r, \Lambda)=\frac{1}{5}\left(e^{-\sqrt{\gamma} r / \Lambda}-1\right)\left(1-e^{-2 r / \Lambda}\right)$, and $\gamma\left(\equiv e^{2} / \Lambda k T_{e}\right)$ is the nonideal-plasma parameter, $c(\gamma) \approx 0.465 \gamma-0.108 \gamma^{2}+0.009 \gamma^{3}$ is the correlation coefficient, and $T_{e}$ is the electron temperature. When $\gamma \rightarrow 0$, i.e., in the case of an ideal plasma, the pseudopotential goes over to the Debye-Hückel potential $V_{D H}(r)=-Z e^{2} e^{-r / \Lambda} / r$. The corresponding force for the pseudopotential $V_{N I}(\mathbf{r})$ is then given by

$$
F(\mathbf{r})=-\frac{Z e^{2} \mathbf{r}}{1+c(\gamma)}\left[\left(\frac{1}{r^{3}}+\frac{1}{r^{2} \Lambda}\right) J(\gamma, r, \Lambda)-\frac{1}{r^{2} \Lambda} K(\gamma, r, \Lambda)\right],
$$

where the functions $J(\gamma, r, \Lambda)$ and $K(\gamma, r, \Lambda)$ are, respectively,

$$
\begin{gathered}
J(\gamma, r, \Lambda)=e^{-r / \Lambda}+\frac{\gamma}{10}\left[-e^{(\sqrt{\gamma}+3) r / \Lambda}+e^{-(\sqrt{\gamma}+1) r / \Lambda}+e^{-3 r / \Lambda}-e^{-r / \Lambda}\right] \\
K(\gamma, r, \Lambda)=\frac{\gamma}{10}\left[(\sqrt{\gamma}+2) e^{-(\sqrt{\gamma}+3) r / \Lambda}-\sqrt{\gamma} e^{-(\sqrt{\gamma}+1) r / \Lambda}-2 e^{-3 r / \Lambda}\right]
\end{gathered}
$$

For the case of electron-ion interactions, i.e., the attractive case, the convenient parametric representation of the hyperbolic-orbit trajectory (Landau and Lifshitz 1975) for the projectile electron $[\mathbf{r}(t)]$ can be represented by

$$
\left.\begin{array}{l}
x=d\left(\varepsilon^{2}-1\right)^{1 / 2} \sinh w, \\
y=d(-\cosh w+\varepsilon), \\
r(t) \equiv|\mathbf{r}(t)|=d(\varepsilon \cosh w-1), \\
t=\frac{d}{v}(\varepsilon \sinh w-w), \quad-\infty<w<\infty,
\end{array}\right\}
$$

where $d, \varepsilon\left(=\sqrt{1+b^{2} / d^{2}}\right)$, and $v$ are half the distance of closest approach in a head-on collision, the eccentricity, and the initial velocity of the projectile electron, respectively. Since the above expression for the hyperbolic-orbit trajectory describes the case of a pure Coulomb field, here we have to modify the parameter $d$ in order to include plasma-screening and collective effects. Including the plasmascreening effect as well as the nonideal-plasma effect, the collision parameter $d$ can be obtained by the perturbation method (Bender and Orszag 1999) with the pseudopotential (6):

$$
d(\gamma, \Lambda) \approx \frac{d_{0}}{1+d_{0} / \Lambda+c(\gamma)}+\frac{d_{0}^{3}}{\Lambda^{2}} \frac{\frac{1}{2}-\frac{1}{5} \gamma^{3 / 2}}{\left[1+d_{0} / \Lambda+c(\gamma)\right]^{3}},
$$

where $d_{0} \equiv Z e^{2} / m v^{2}$. After some algebra, the Fourier coefficients of the force are 
found to be

$$
F_{\mu \omega}=-\frac{Z e^{2}}{2 \pi \bar{v} a_{Z}^{2}} \bar{F}_{\mu \omega} \quad(\mu=x, y),
$$

where $a_{Z}\left(\equiv a_{0} / Z=\hbar^{2} / Z m e^{2}\right)$ is the first Bohr radius of the hydrogenic ion with nuclear charge $Z, m$ is the electron rest mass, and $\bar{v} \equiv v / a_{Z}$. Here, the $x$ and $y$ components of the scaled Fourier coefficient $\left(\bar{F}_{x \omega}, \bar{F}_{y \omega}\right)$ are given by

$$
\begin{gathered}
\bar{F}_{x \omega}=\frac{\bar{d}^{2}}{1+c(\gamma)} \int_{-\infty}^{\infty} d w(\varepsilon \cosh w-1)\left(\varepsilon^{2}-1\right)^{1 / 2} \sinh w e^{i \xi(\varepsilon \sinh w-w)} \\
\times\left\{\left[\frac{1}{\bar{d}^{3}(\varepsilon \cosh w-1)^{3}}+\frac{a_{\Lambda}}{\bar{d}^{2}(\varepsilon \cosh w-1)^{2}}\right] J\left(\gamma, \varepsilon, \bar{d}, a_{\Lambda}\right)\right. \\
\left.\quad-\frac{a_{\Lambda}}{\bar{d}^{2}(\varepsilon \cosh w-1)^{2}} K\left(\gamma, \varepsilon, \bar{d}, a_{\Lambda}\right)\right\}, \\
\bar{F}_{y \omega}=\frac{\bar{d}^{2}}{1+c(\gamma)} \int_{-\infty}^{\infty} d w(\varepsilon \cosh w-1)(-\cosh w+\varepsilon) e^{i \xi(\varepsilon \sinh w-w)} \\
\times\left\{\left[\frac{1}{\bar{d}^{3}(\varepsilon \cosh w-1)^{3}}+\frac{a_{\Lambda}}{\bar{d}^{2}(\varepsilon \cosh w-1)^{2}}\right] J\left(\gamma, \varepsilon, \bar{d}, a_{\Lambda}\right)\right. \\
\left.-\frac{a_{\Lambda}}{\bar{d}^{2}(\varepsilon \cosh w-1)^{2}} K\left(\gamma, \varepsilon, \bar{d}, a_{\Lambda}\right)\right\},
\end{gathered}
$$

respectively, where $\bar{d}\left(\equiv d / a_{Z}\right)$ is the scaled collision parameter, $\xi \equiv \omega d / v, a_{\Lambda}$ ( $\left.\equiv a_{Z} / \Lambda\right)$ is the reciprocal scaled Debye length, and $\bar{b}\left(\equiv b / a_{Z}\right)$ is the scaled impact parameter. Here, the functions $J\left(\gamma, \varepsilon, \bar{d}, a_{\Lambda}\right)$ and $K\left(\gamma, \varepsilon, \bar{d}, a_{\Lambda}\right)$ are, respectively,

$$
\begin{aligned}
J\left(\gamma, \varepsilon, \bar{d}, a_{\Lambda}\right)= & e^{-a_{\Lambda} \bar{d}(\varepsilon \cosh w-1)}+\frac{1}{10} \gamma\left[-e^{-a_{\Lambda}(\sqrt{\gamma}+3) \bar{d}(\varepsilon \cosh w-1)}\right. \\
& \left.+e^{-a_{\Lambda}(\sqrt{\gamma}+1) \bar{d}(\varepsilon \cosh w-1)}+e^{-3 a_{\Lambda} \bar{d}(\varepsilon \cosh w-1)}-e^{-a_{\Lambda} \bar{d}(\varepsilon \cosh w-1)}\right], \\
K\left(\gamma, \varepsilon, \bar{d}, a_{\Lambda}\right)= & \frac{1}{10} \gamma\left[(\sqrt{\gamma}+2) e^{-a_{\Lambda}(\sqrt{\gamma}+3) \bar{d}(\varepsilon \cosh w-1)}-\sqrt{\gamma} e^{-a_{\Lambda}(\sqrt{\gamma}+1) \bar{d}(\varepsilon \cosh w-1)}\right. \\
& \left.-2 e^{-3 a_{\Lambda} \bar{d}(\varepsilon \cosh w-1)}\right] .
\end{aligned}
$$

In the nonrelativistic limit, the classical bremsstrahlung cross-section is found to be

$$
d \sigma_{b}=\frac{4}{3} \frac{\alpha^{3} a_{0}^{2}}{\bar{E}_{e}} \frac{d \omega}{\omega} \int_{\bar{b}_{\min }}^{\bar{b}_{\max }} d \bar{b} \bar{b}\left(\left|\bar{F}_{x \omega}\right|^{2}+\left|\bar{F}_{y \omega}\right|^{2}\right),
$$

where $\alpha\left(=e^{2} / \hbar c \approx \frac{1}{137}\right)$ is the fine-structure constant, $\bar{E}_{e}\left(\equiv m v^{2} / 2 Z^{2} R y\right)$ is the scaled kinetic energy of the projectile electron, and $R y\left(=m e^{4} / 2 \hbar^{2} \approx 13.6 \mathrm{eV}\right)$ is the Rydberg constant. Here, the minimum scaled impact parameter $\bar{b}_{\text {min }}$ corresponds to the closest distance of approach at which the electrostatic potential energy of interaction is equal to the maximum possible energy transfer:

$$
2 m v^{2}=\frac{Z e^{2}}{b} e^{-b / \Lambda} \frac{1+\frac{1}{2} \gamma f(\gamma, b, \Lambda)}{1+c(\gamma)} .
$$

The maximum scaled impact parameter $\bar{b}_{\max }$ is determined by the screening length for the plasma: $\bar{b}_{\max } \approx a_{\Lambda}^{-1}$. 


\section{Bremsstrahlung radiation cross-section}

Using (17), the differential bremsstrahlung radiation cross-section (Jackson 1999) is defined by

$$
\begin{aligned}
\frac{d \chi_{b}}{d E_{p}} & \equiv \frac{d \sigma_{b}}{\hbar d \omega} \hbar \omega \\
& =\frac{4}{3} \frac{\alpha^{3} a_{0}^{2}}{\bar{E}_{e}} \int_{\bar{b}_{\min }}^{\bar{b}_{\max }} d \bar{b} \bar{b}\left(\left|\bar{F}_{x \omega}\right|^{2}+\left|\bar{F}_{y \omega}\right|^{2}\right),
\end{aligned}
$$

where $E_{p}(\equiv \hbar \omega)$ is the energy of the radiation photon. In the nonrelativistic limit, the parameter $\xi$ can be expressed as $\xi=\bar{E}_{p} \bar{d} / 2 \sqrt{\bar{E}_{e}}$ where $\bar{E}_{p}\left(\equiv \hbar \omega / Z^{2} R y\right)$ is the scaled radiation photon energy. Using the screened hyperbolic-orbit trajectory method, the scaled doubly differential bremsstrahlung radiation (SDDBR) crosssection, i.e., the scaled differential bremsstrahlung radiation cross-section per scaled impact parameter, is found to be

$$
\frac{d^{2} \chi_{b}}{d \bar{E}_{p} d \bar{b}} / \pi a_{0}^{2}=\frac{4}{3} \frac{\alpha^{3}}{\pi \bar{E}_{e}} \bar{b}\left[\left|\bar{F}_{x \omega}\left(\gamma, \bar{b}, a_{\Lambda}, \bar{E}_{p}, \bar{E}_{e}\right)\right|^{2}+\left|\bar{F}_{y \omega}\left(\gamma, \bar{b}, a_{\Lambda}, \bar{E}_{p}, \bar{E}_{e}\right)\right|^{2}\right],
$$

where the Fourier coefficients $\bar{F}_{x \omega}$ and $\bar{F}_{y \omega}$ are given in (13) and (14). The dependences of the SDDBR cross-section on the plasma-screening and collective effects have been explicitly indicated through the scaled reciprocal Debye length $a_{\Lambda}$ and the nonideal-plasma parameter $\gamma$.

In order to investigate the plasma-screening effects as well as the nonideal-plasma effects on the bremsstrahlung radiation cross-section, we consider two cases of the ratio of the radiation photon energy to the kinetic energy of the projectile electron, namely, $E_{p} / E_{e}\left(=2 \hbar \omega / m v^{2}\right)=0.1$ (i.e., a soft-photon case) and 0.9 (i.e., a hard-photon case), and we consider three cases of the nonideal-plasma parameter, namely, $\gamma=1,0.1$, and 0 . Here, we choose $a_{\Lambda}=0.1$ and $\bar{E}_{e}=0.9$, since the classical-trajectory approximation is known to be reliable for low projectile energies $(v<Z \alpha c)$ (Melia 2001). Figure 1 shows the SDDBR cross-sections (21) for electron-ion Coulomb collisions in ideal $(\gamma=0)$ and nonideal $(\gamma \neq 0)$ plasmas. As we can see from this figure, the bremsstrahlung radiation cross-section in an ideal plasma described by the Debye-Hückel potential is found to be greater than that in a nonideal plasma, i.e., the collective effects reduce the bremssstrahlung radiation cross-sections in both the soft- and hard-photon cases. However, for small impact parameters, the nonideal-plasma effect on the bremsstrahlung cross-section is found to be quite small. Table 1 shows the maximum values of the differential bremsstrahlung cross-sections for $\gamma=1,0.1$, and 0 . It should be noted that the nonideal-plasma effect on the electron-ion bremsstrahlung process is more significant in the hard-photon radiation case $(\approx 14.8 \%)$ than that in the soft-photon radiation case $(\approx 8.8 \%)$. The maximum positions are also indicated in the table in parentheses. It is also found that the maximum position of the bremsstrahlung cross-section gets closer to the target ion with increasing nonideal-plasma effect.

\section{Conclusions}

We have investigated plasma-screening and collective effects on the electron-ion Coulomb bremsstrahlung process in a classical nonideal plasma. An effective pseudopotential model has been applied to describe the electron-ion interaction potential, 
Table 1. The maximum values of the scaled doubly differential bremsstrahlung radiation cross-sections $d^{2} \chi_{b} / d \bar{E}_{p} d \bar{b}$ in units of $\pi a_{0}^{2}$ for both the soft- and hard-photon radiation cases when $a_{\Lambda}=0.1$ and $\bar{E}_{e}=0.9$. (The maximum positions are indicated in parentheses.)

\begin{tabular}{ccc}
\hline & $d^{2} \chi_{b} / d \bar{E}_{p} d \bar{b}$ & $d^{2} \chi_{b} / d \bar{E}_{p} d \bar{b}$ \\
& $\left(E_{p} / E_{e}=0.1\right.$ : soft-photon $)$ & $\left(E_{p} / E_{e}=0.9\right.$ : hard-photon $)$ \\
\hline$\gamma=1$ & $7.160 \times 10^{-7}$ & $9.494 \times 10^{-7}$ \\
& $(\bar{b}=0.41)$ & $(\bar{b}=0.44)$ \\
$\gamma=0.1$ & $7.629 \times 10^{-7}$ & $1.074 \times 10^{-6}$ \\
& $(\bar{b}=0.51)$ & $(\bar{b}=0.54)$ \\
$\gamma=0$ & $7.847 \times 10^{-7}$ & $1.114 \times 10^{-6}$ \\
& $(\bar{b}=0.52)$ & $(\bar{b}=0.56)$ \\
\hline
\end{tabular}
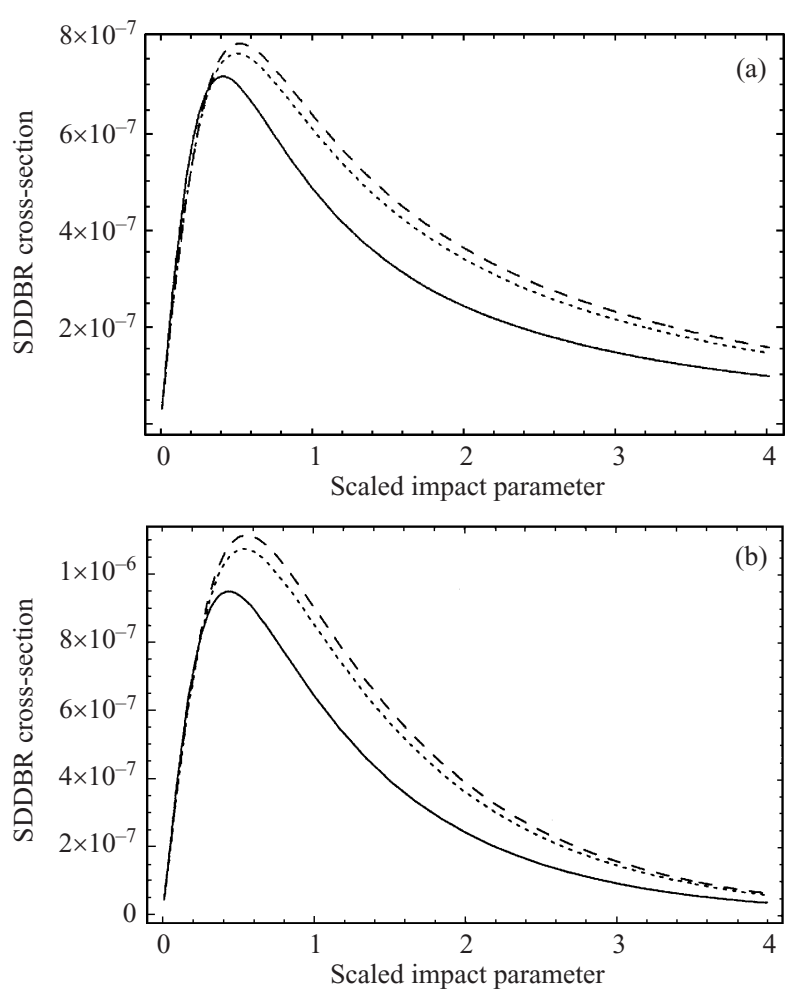

Figure 1. The scaled doubly differential bremsstrahlung radiation cross-section $d^{2} \chi_{b} / d \bar{E}_{p} d \bar{b}$ in units of $\pi a_{0}^{2}$ as a function of the scaled impact parameter $\left(\bar{b}=b / a_{Z}\right)$ for $a_{\Lambda}=0.1$ and $\bar{E}_{e}=0.9$ : (a) $E_{p} / E_{e}=0.1$ (soft-photon); (b) $E_{p} / E_{e}=0.9$ (hard-photon);,$- \gamma=1$; $\cdots \cdots, \gamma=0.1 ;---, \gamma=0$.

including plasma-screening as well as collective effects in a nonideal plasma. The screened hyperbolic-orbit trajectory method has been applied to describe the motion of the projectile electron in a nonideal plasma and to visualize the variation of the differential bremsstrahlung radiation cross-section as a function of the scaled impact parameter $b$, eccentricity $\varepsilon$, nonideal-plasma parameter $\gamma$, Debye length $\Lambda$, kinetic energy of the projectile electron $E_{e}$, and radiation-photon energy $E_{p}$. The results show that the bremsstrahlung radiation cross-section for electron-ion 
Coulomb collisions in an ideal plasma described by the Debye-Hückel model is always greater than that in a nonideal plasma, i.e., the collective effect $(\gamma \neq 0)$ suppresses the bremsstrahlung radiation cross-sections for both the soft- and hardphoton radiation cases. It is also found that the nonideal-plasma effect on the bremsstrahlung process in the hard-photon radiation case is more significant than in the soft-photon radiation case. For small impact parameters, it is found that the nonideal-plasma effect on the bremsstrahlung cross-section is quite small. The maximum position of the bremsstrahlung radiation cross-section gets closer to the target ion with increasing nonideal-plasma parameter. These results provide useful information on the Coulomb bremsstrahlung process in a classical nonideal plasma.

\section{Acknowledgements}

One of the authors (Y.-D. Jung) gratefully acknowledges Professor R. J. Gould and Professor T. M. O'Neil for warm hospitality while visiting the University of California, San Diego. He also thanks Professor B. Talin, Professor R. Sauerbrey, and Dr M. Rosenberg for useful discussions on nonideal plasmas. This work was supported by the Korean Ministry of Education through the Brain Korea (BK21) Project and by the Korea Basic Science Institute through the HANBIT User Development Program (FY2002).

\section{References}

Arkhipov, Yu. V., Baimbetov, F. B. and Davletov, A. E. 2000a Eur. Phys. J. D8, 299

Arkhipov, Yu. V., Baimbetov, F. B., Davletov, A. E. and Starikov. K. V. 2000b Plasma Phys. Contr. Fusion 42, 455.

Arkhipov, Yu. V., Baimbetov, F. B., Davletov, A. E. and Starikov, K. V. 2001 Physica Scripta 63, 194.

Baimbetov, F. B., Nurekenov, Kh. T. and Ramazanov, T. S. 1995 Phys. Lett. 202A, 211.

Bekefi, G. 1966 Radiation Processes in Plasmas. New York: Wiley.

Bender, C. M. and Orszag, S. A. 1999 Advanced Mathematical Methods for Scientists and Engineers. New York: Springer-Verlag.

Bethe, H. A. and Salpeter, E. E. 1957 Quantum Mechanics of One-and Two-Electron Atoms. Berlin: Springer-Verlag.

Gould, R. J. 1970 Am. J. Phys. 38, 189.

Gould, R. J. 1980 Astrophys. J. 238, 1026.

Gould, R. J. 1990 Astrophys. J. 362, 284.

Hutchinson, I. H. 1987 Principles of Plasma Diagnostics. Cambridge University Press.

Jackson, J. D. 1999 Classical Electrodynamics, 3rd edn. New York: Wiley.

Jung, Y.-D. 1994 Phys. Plasmas 1, 785.

Jung, Y.-D. 1997 Phys. Rev. E55, 21.

Jung, Y.-D. and Yang, K. S. 1997 Astrophys. J. 479, 912.

Landau, L. D. and Lifshitz, E. M. 1975 The Classical Theory of Fields, 4th edn. Oxford: Pergamon.

Melia, F. 2001 Electrodynamics. University of Chicago Press.

Padmanabhan, T. 2001 Theoretical Astrophysics, Vol. II: Stars and Stellar Systems. Cambridge University Press.

Tsytovich, V. N. 1995 Lectures on Non-Linear Plasma Kinetics. Berlin: Springer-Verlag. 